\title{
THE F. AND M. RIESZ THEOREM ${ }^{1}$
}

\section{FRANK FORELLI}

ABSTRACT. We weaken the hypothesis of the F. and M. Riesz theorem.

1. If $X$ is a locally compact Hausdorff space, then we will denote by $M_{+}(X)$ the class of all Radon measures on $X$. Thus if $\mu \in M_{+}(X)$ and $E \subset X$, then $\mu(E) \geq 0$. We will denote by $M(X)$ the complex linear span of those $\mu$ in $M_{+}(X)$ for which $\mu(X)<\infty$. If $X$ is a locally compact abelian group, and if $\mu \in M(X)$, then we will denote (as is usual) by $\hat{\mu}$ the Fourier transform of $\mu$. We let $\mathbf{Z}_{+}=\{k: k \in \mathbf{Z}, k>0\}$. We will denote by $\gamma$ the Lebesgue measure on R. The well-known F. and M. Riesz theorem (in one of its many versions) states that if $\mu \in M(R)$ and if $\hat{\mu}=0$ on $(0, \infty)$, then $\mu \ll \gamma$. The purpose of this paper is to point out that the condition on $\hat{\mu}$ may be weakened as follows.

2. Theorem. ${ }^{2}$ Let $a$ and $b$ in $(0, \infty)$ be linearly independent over $\mathbf{Z}$, and let $S=\left\{j a+k b:(j, k) \in \mathbf{Z}_{+} \times \mathbf{Z}_{+}\right\}$. If $\mu \in M(\mathbf{R})$ and if $\hat{\mu}=0$ on $S$, then $\mu<\gamma$.

3. With regard to Theorem 2 we remark that if $t>0$, then $(0, t) \cap S$ is finite.

4. We will now prove Theorem 2. We define $\phi: \mathbf{R} \rightarrow \mathbf{T} \times \mathbf{T}$ by $\phi(t)=$ $\left(e^{i a t}, e^{i b t}\right)$ and we let $\lambda=\mu \circ \phi^{-1}$. Thus $\lambda \in M(\mathbf{T} \times \mathbf{T})$. If $f \in C(\mathbf{T} \times \mathbf{T})$, then $\int f d \lambda=\int f \circ \phi d \mu$; hence if $(j, k) \in \mathbf{Z} \times \mathbf{Z}$, then

$$
\hat{\lambda}(j, k)=\int \bar{z}^{j} \bar{w}^{k} d \lambda(z, w)=\int e^{-i(j a+k b) t} d \mu(t)=\hat{\mu}(j a+k b) .
$$

Thus $\hat{\lambda}=0$ on $\mathbf{Z}_{+} \times \mathbf{Z}_{+}$; hence by [1, Theorem 6.2.2], if $E \subset \mathbf{R}$ and $\gamma(E)=0$, then $\lambda(\phi(E))=0$. Since $E=\phi^{-1}(\phi(E))$, we have $\mu(E)=0$ which completes the proof of Theorem 2 .

\section{REFERENCE}

1. W. Rudin, Function theory in polydiscs, Benjamin, New York, 1969. MR 41 \#501.

DEPARTMENT OF MATHEMATICS, UNIVERSITY OF WISCONSIN, MADISON, WISCONSIN 53706

Received by the editors March 18, 1974.

AMS (MOS) subject classifications (1970). Primary $42 \mathrm{~A} 68$.

${ }^{1}$ Research supported by NSF GP 33897X1 (144-C918).

2 The referee reports that Theorem 2 has also been obtained by Brian Cole. 